\title{
Repurposing Acitretin as an Antipseudomonal Agent Targeting the Pseudomonas aeruginosa Iron- regulated Heme Oxygenase
}

Elizabeth A. Robinson ${ }^{1}$, Angela Wilks ${ }^{1, *}$, Fengtian Xue ${ }^{1, *}$

${ }^{1}$ Department of Pharmaceutical Sciences, University of Maryland Baltimore, Baltimore MD 21201, United States

*Corresponding Authors:

Angela Wilks - Department of Pharmaceutical Sciences, University of Maryland School of Pharmacy, 20

Penn Street, Baltimore, Maryland 21201; 410-706-2537; Email: awilks@,rx.umaryland.edu

Fengtian Xue - Department of Pharmaceutical Sciences, University of Maryland School of Pharmacy, 20

Penn Street, Baltimore, Maryland 21201; 410-706-8521; ORCID: 0000-0002-4132-9887; Email:

fxue@,rx.umaryland.edu

\section{Table of Contents:}

Figure S1. HemO and hHO-1 binding heme Page S2

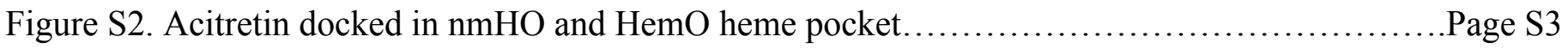

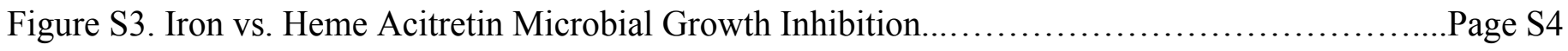

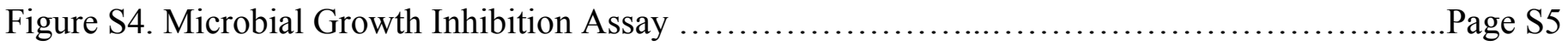

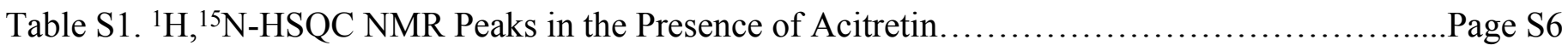



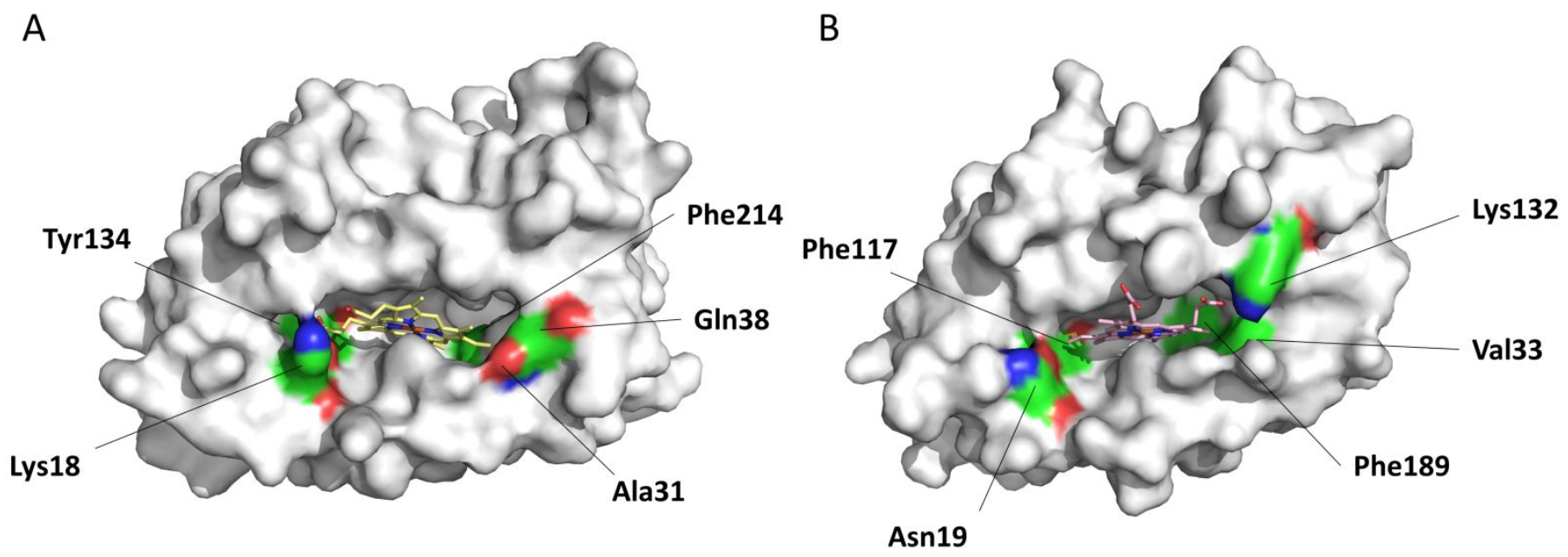

Figure S1 HemO and hHO-1 binding heme. Graphical representations of hHO-1 (PDB 1N45) and HemO (PDB 1SK7) with heme bound. (A) hHO-1 and (B) HemO shown with heme binding residues labeled and highlighted. 

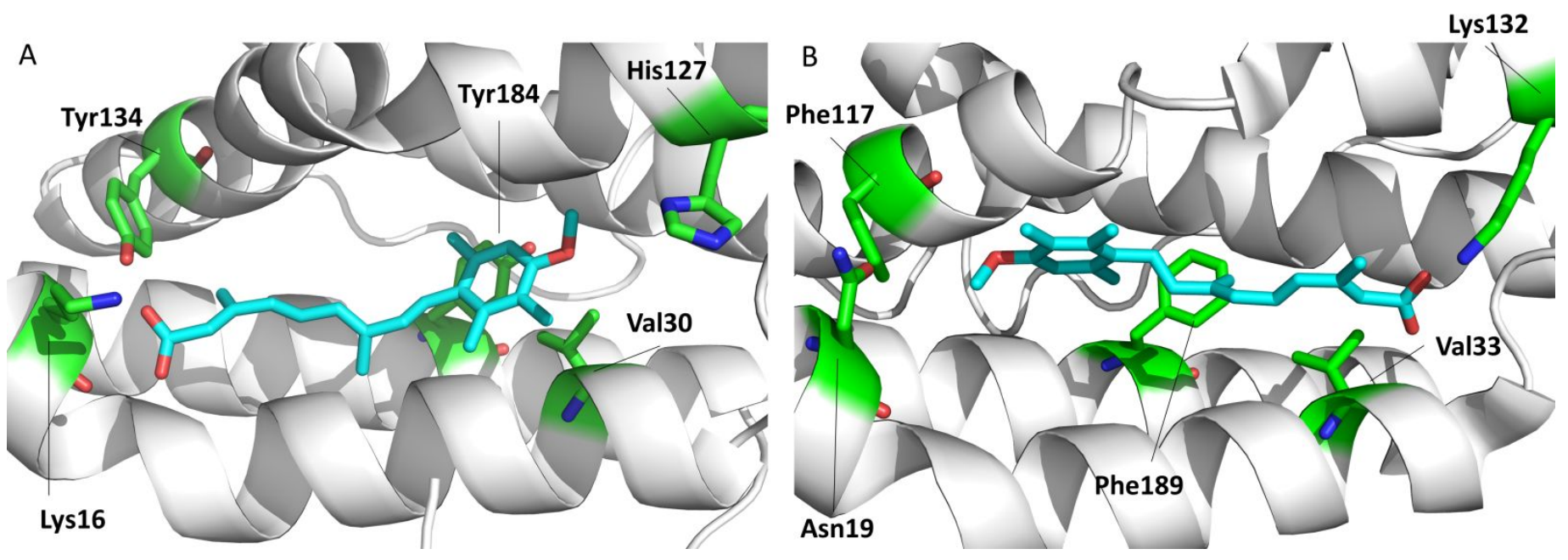

Figure S2. Acitretin docked in HemO and nmHO heme pocket. Graphical representations of HemO (PDB 1SK7) and nmHO (PDB 1J77) with acitretin docked within the heme pocket using AutoDock Vina. (A) HemO and (B) nmHO shown with heme binding residues labeled and highlighted. 
A

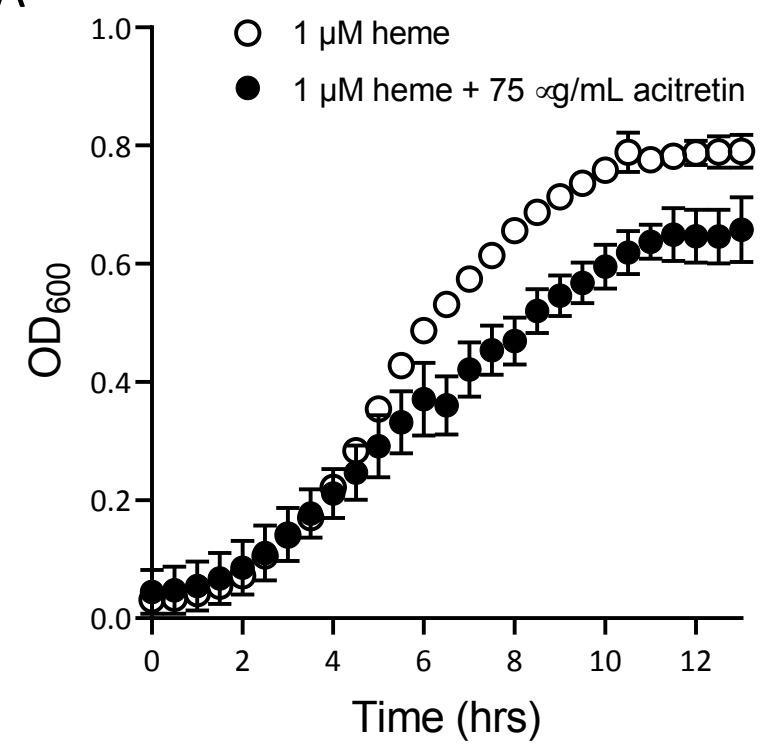

B

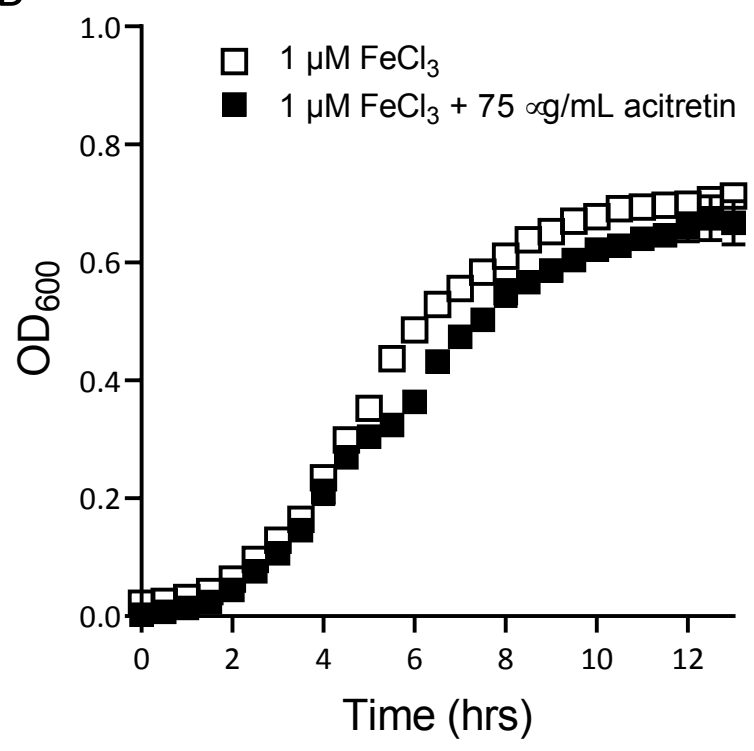

Figure S3 Iron vs. Heme Acitretin Microbial Growth Inhibition. Acitretin concentration dependent minimal inhibitory growth assays performed in minimal media supplemented with either $1 \mu \mathrm{M}$ heme (A) or $1 \mu \mathrm{M} \mathrm{FeCl}_{3}$ (B) with PAO1 grown for 13 hours. Acitretin concentration $(75 \mu \mathrm{g} / \mathrm{mL})$ was supplemented in three biological replicates. Each point on the graph represents the average of triplicate measurements and is shown with a one standard deviation error bar. 

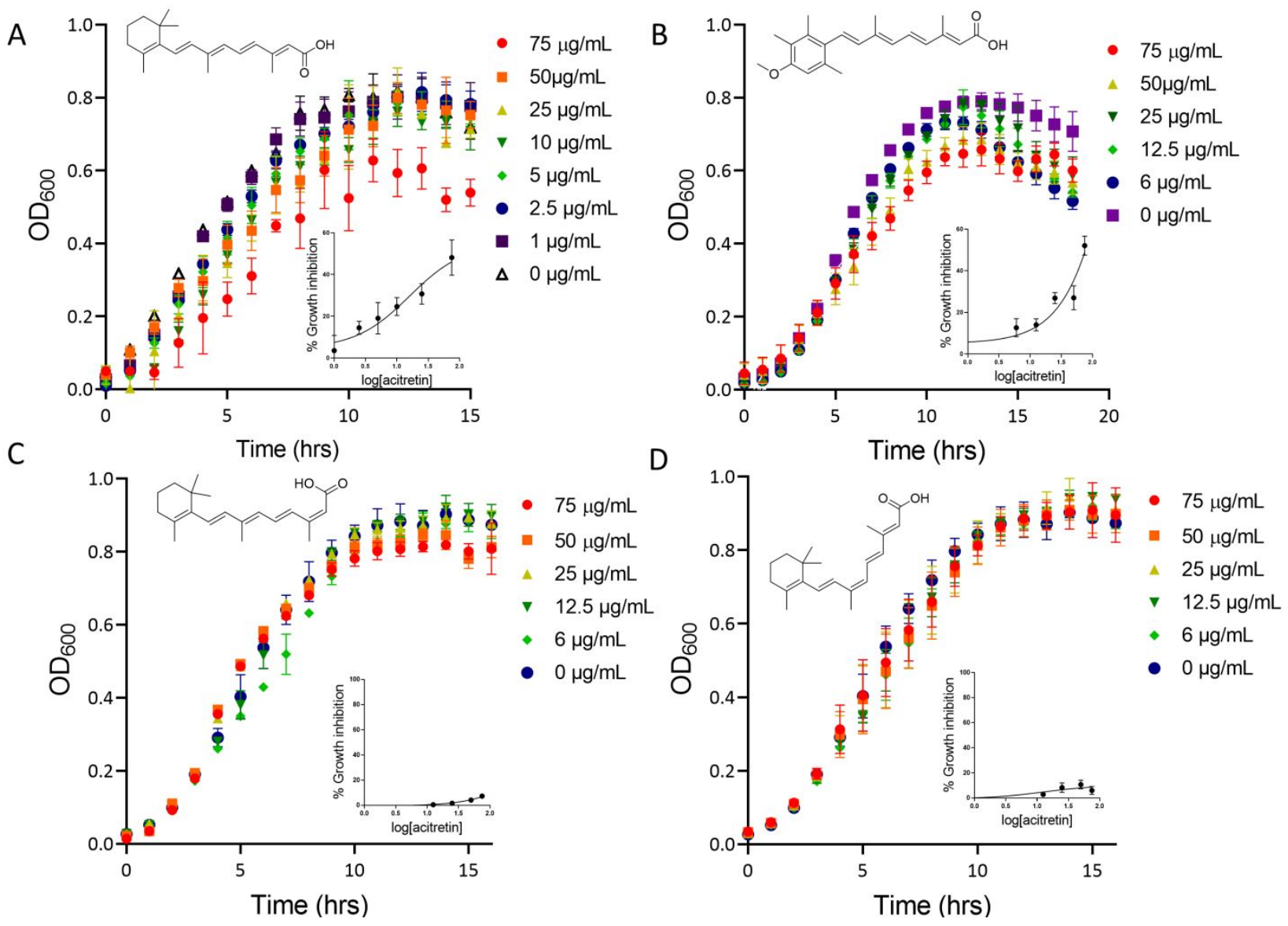

Figure S4 Microbial Growth Inhibition Assay. Dose-dependent curves of retinoic acid derivatives concentration dependent minimal inhibitory growth assays performed in minimal media supplemented with 1 $\mu \mathrm{M}$ heme with PAO1 grown for 15-20 h. Retinoic acid (A), acitretin (B), 13-cis retinoic acid (C), and 9-cis retinoic acid (D) concentrations were supplemented in three biological replicates. Each point on the graph represents the average of triplicate measurements and is shown with a one standard deviation error bar. 
Table S1. ${ }^{1} \mathrm{H},{ }^{15} \mathrm{~N}-\mathrm{HSQC}$ NMR Peaks in the Presence of Acitretin

Apo-HemO

\begin{tabular}{|c|c|c|c|c|c|c|c|}
\hline Peak & HN (ppm) & N (ppm) & HN (ppm) & N (ppm) & $\Delta H N(p p m)$ & $\Delta N(p p m)$ & \\
\hline $\mathrm{aq}$ & 8.868 & 123.5 & 8.854 & 123.4 & 0.014 & 0.1 & 0.024413 \\
\hline by & 7.82 & 122.4 & 7.812 & 122.4 & 0.008 & 0 & 0.008 \\
\hline dg & 7.811 & 119.7 & 7.811 & 119.7 & 0 & 0 & 0 \\
\hline ed & 8.166 & 115.3 & 8.175 & 115.2 & 0.009 & 0.1 & 0.021932 \\
\hline$f x$ & 8.238 & 122.7 & 8.223 & 122.7 & 0.015 & 0 & 0.015 \\
\hline gj & 7.781 & 119.7 & 7.784 & 119.8 & 0.003 & 0.1 & 0.020224 \\
\hline hk & 8.328 & 120.9 & 8.328 & 121 & 0 & 0.1 & 0.02 \\
\hline hl & 8.801 & 116.6 & 8.808 & 116.5 & 0.007 & 0.1 & 0.02119 \\
\hline hz & 7.719 & 117.6 & 7.718 & 117.5 & 0.001 & 0.1 & 0.020025 \\
\hline ih & 7.364 & 118 & 7.36 & 118 & 0.004 & 0 & 0.004 \\
\hline it & 7.921 & 119.8 & 7.921 & 119.8 & 0 & 0 & 0 \\
\hline jb & 7.966 & 118.3 & 7.945 & 118.3 & 0.021 & 0 & 0.021 \\
\hline jc & 7.993 & 118.3 & 7.978 & 118.4 & 0.015 & 0.1 & 0.025 \\
\hline jw & 8.471 & 122.1 & 8.466 & 122 & 0.005 & 0.1 & 0.020616 \\
\hline ke & 9.083 & 119.9 & 9.064 & 120 & 0.019 & 0.1 & 0.027586 \\
\hline T003 & 8.257 & 114.8 & 8.264 & 115 & 0.007 & 0.2 & 0.040608 \\
\hline L004 & 8.329 & 124.4 & 8.338 & 124.5 & 0.009 & 0.1 & 0.021932 \\
\hline A005 & 8.304 & 126.3 & 8.315 & 126.3 & 0.011 & 0 & 0.011 \\
\hline N012 & 8.435 & 119.1 & 8.403 & 119.3 & 0.032 & 0.2 & 0.051225 \\
\hline L013 & 8.066 & 121.2 & 8.069 & 121.2 & 0.003 & 0 & 0.003 \\
\hline R014 & 9.072 & 128.3 & 9.067 & 128.3 & 0.005 & 0 & 0.005 \\
\hline Q016 & 6.884 & 121.4 & 6.88 & 121.5 & 0.004 & 0.1 & 0.020396 \\
\hline R017 & 8.416 & 122.1 & 8.402 & 122.2 & 0.014 & 0.1 & 0.024413 \\
\hline L018 & 9.189 & 118.3 & 9.173 & 118.3 & 0.016 & 0 & 0.016 \\
\hline N019 & 7.836 & 119.4 & 7.87 & 119.7 & 0.034 & 0.3 & 0.068964 \\
\hline L020 & 7.501 & 122.6 & 7.499 & 123 & 0.002 & 0.4 & 0.080025 \\
\hline L021 & 8.623 & 118.6 & 8.609 & 118.2 & 0.014 & 0.4 & 0.081216 \\
\hline T022 & 7.733 & 105.7 & 7.733 & 105.7 & 0 & 0 & 0 \\
\hline L029 & 8.046 & 121.9 & 8.069 & 121.8 & 0.023 & 0.1 & 0.03048 \\
\hline L032 & 8.198 & 126 & 8.212 & 126 & 0.014 & 0 & 0.014 \\
\hline S035 & 8.026 & 115.2 & 8.046 & 115.2 & 0.02 & 0 & 0.02 \\
\hline K036 & 7.502 & 119.4 & 7.502 & 119.4 & 0 & 0 & 0 \\
\hline E037 & 7.589 & 110.7 & 7.601 & 110.9 & 0.012 & 0.2 & 0.041761 \\
\hline A040 & 7.901 & 120.7 & 7.948 & 120.8 & 0.047 & 0.1 & 0.051078 \\
\hline S041 & 7.547 & 104.7 & 7.547 & 104.7 & 0 & 0 & 0 \\
\hline D043 & 8.309 & 118.3 & 8.299 & 118.1 & 0.01 & 0.2 & 0.041231 \\
\hline N044 & 8.073 & 121 & 8.084 & 120.9 & 0.011 & 0.1 & 0.022825 \\
\hline F045 & 8.647 & 124 & 8.647 & 124 & 0 & 0 & 0 \\
\hline A046 & 8.828 & 121.2 & 8.843 & 121 & 0.015 & 0.2 & 0.04272 \\
\hline F048 & 7.741 & 124.5 & 7.688 & 124.2 & 0.053 & 0.3 & 0.080056 \\
\hline
\end{tabular}




\begin{tabular}{|c|c|c|c|c|c|c|c|}
\hline V049 & 8.88 & 121.4 & 8.901 & 121.5 & 0.021 & 0.1 & 0.029 \\
\hline A051 & 7.248 & 120.9 & 7.28 & 120.7 & 0.032 & 0.2 & 0.051225 \\
\hline Q052 & 7.97 & 117.4 & 7.986 & 117.2 & 0.016 & 0.2 & 0.043081 \\
\hline Y053 & 9.264 & 120.1 & 9.255 & 120.2 & 0.009 & 0.1 & 0.021932 \\
\hline L054 & 8.318 & 120.3 & 8.33 & 120.3 & 0.012 & 0 & 0.012 \\
\hline F055 & 8.725 & 123.3 & 8.684 & 123.3 & 0.041 & 0 & 0.041 \\
\hline Q056 & 8.351 & 115.9 & 8.351 & 115.9 & 0 & 0 & 0 \\
\hline D058 & 7.817 & 121.4 & 7.826 & 121 & 0.009 & 0.4 & 0.080505 \\
\hline L059 & 6.932 & 113.5 & 6.937 & 113.6 & 0.005 & 0.1 & 0.020616 \\
\hline E060 & 7.393 & 123 & 7.404 & 122.9 & 0.011 & 0.1 & 0.022825 \\
\hline L062 & 7.713 & 116.6 & 7.707 & 116.8 & 0.006 & 0.2 & 0.040447 \\
\hline Y063 & 7.672 & 114.8 & 7.663 & 114.7 & 0.009 & 0.1 & 0.021932 \\
\hline R064 & 7.386 & 112.3 & 7.386 & 112.3 & 0 & 0 & 0 \\
\hline N065 & 7.303 & 122.5 & 7.324 & 122.5 & 0.021 & 0 & 0.021 \\
\hline E066 & 9.208 & 128.6 & 9.192 & 128.5 & 0.016 & 0.1 & 0.025612 \\
\hline A067 & 8.035 & 122.3 & 8.035 & 122.1 & 0 & 0.2 & 0.04 \\
\hline R070 & 7.403 & 114.8 & 7.42 & 114.8 & 0.017 & 0 & 0.017 \\
\hline L071 & 7.213 & 118.8 & 7.239 & 118.8 & 0.026 & 0 & 0.026 \\
\hline F072 & 7.96 & 115.3 & 7.958 & 115.3 & 0.002 & 0 & 0.002 \\
\hline L075 & 8.07 & 123.4 & 8.054 & 123.3 & 0.016 & 0.1 & 0.025612 \\
\hline A076 & 8.734 & 118.3 & 8.715 & 118.3 & 0.019 & 0 & 0.019 \\
\hline S077 & 7.999 & 112.2 & 7.993 & 112.2 & 0.006 & 0 & 0.006 \\
\hline A079 & 7.086 & 122.4 & 7.115 & 122.2 & 0.029 & 0.2 & 0.049406 \\
\hline R080 & 7.922 & 118.5 & 7.939 & 118.6 & 0.017 & 0.1 & 0.026249 \\
\hline D082 & 8.263 & 120.2 & 8.259 & 120.3 & 0.004 & 0.1 & 0.020396 \\
\hline A083 & 8.218 & 123.7 & 8.215 & 123.6 & 0.003 & 0.1 & 0.020224 \\
\hline A084 & 8.257 & 118 & 8.253 & 117.9 & 0.004 & 0.1 & 0.020396 \\
\hline R085 & 8.154 & 117.3 & 8.125 & 117.2 & 0.029 & 0.1 & 0.035228 \\
\hline A086 & 7.865 & 123.3 & 7.872 & 123.3 & 0.007 & 0 & 0.007 \\
\hline D087 & 8.383 & 121.8 & 8.394 & 121.8 & 0.011 & 0 & 0.011 \\
\hline L088 & 7.897 & 119.1 & 7.92 & 119 & 0.023 & 0.1 & 0.03048 \\
\hline A089 & 7.504 & 120.7 & 7.498 & 120.6 & 0.006 & 0.1 & 0.020881 \\
\hline L091 & 7.657 & 115.9 & 7.667 & 115.8 & 0.01 & 0.1 & 0.022361 \\
\hline G092 & 8.062 & 109.3 & 8.068 & 109.3 & 0.006 & 0 & 0.006 \\
\hline E097 & 8.523 & 119.8 & 8.537 & 119.7 & 0.014 & 0.1 & 0.024413 \\
\hline G098 & 8.585 & 111.7 & 8.591 & 111.6 & 0.006 & 0.1 & 0.020881 \\
\hline D099 & 8.704 & 120.5 & 8.716 & 120.6 & 0.012 & 0.1 & 0.023324 \\
\hline S101 & 8.828 & 117 & 8.838 & 117 & 0.01 & 0 & 0.01 \\
\hline V102 & 8.323 & 123 & 8.341 & 123 & 0.018 & 0 & 0.018 \\
\hline R103 & 8.326 & 122.1 & 8.354 & 122.1 & 0.028 & 0 & 0.028 \\
\hline A105 & 7.479 & 123.2 & 7.455 & 122.9 & 0.024 & 0.3 & 0.064622 \\
\hline D106 & 8.469 & 120.9 & 8.466 & 120.8 & 0.003 & 0.1 & 0.020224 \\
\hline L107 & 7.871 & 121.8 & 7.903 & 121.7 & 0.032 & 0.1 & 0.037736 \\
\hline S108 & 8.534 & 118.7 & 8.581 & 118.9 & 0.047 & 0.2 & 0.061717 \\
\hline
\end{tabular}




\begin{tabular}{|c|c|c|c|c|c|c|c|}
\hline L109 & 8.691 & 123.2 & 8.675 & 123 & 0.016 & 0.2 & 0.043081 \\
\hline A110 & 8.498 & 117.4 & 8.479 & 117.2 & 0.019 & 0.2 & 0.044283 \\
\hline E111 & 7.57 & 115.5 & 7.529 & 115.4 & 0.041 & 0.1 & 0.045618 \\
\hline A112 & 8.736 & 121.4 & 8.763 & 121.3 & 0.027 & 0.1 & 0.033601 \\
\hline L113 & 8.299 & 116.3 & 8.336 & 116.3 & 0.037 & 0 & 0.037 \\
\hline G114 & 7.837 & 104.9 & 7.837 & 104.9 & 0 & 0 & 0 \\
\hline W115 & 7.77 & 122.2 & 7.75 & 122 & 0.02 & 0.2 & 0.044721 \\
\hline F117 & 8.79 & 120.5 & 8.84 & 120.2 & 0.05 & 0.3 & 0.078102 \\
\hline V118 & 7.593 & 120.2 & 7.681 & 120.3 & 0.088 & 0.1 & 0.090244 \\
\hline S119 & 8.505 & 114.8 & 8.505 & 114.8 & 0 & 0 & 0 \\
\hline E120 & 9.483 & 118.5 & 9.452 & 118.6 & 0.031 & 0.1 & 0.036892 \\
\hline G121 & 8.377 & 111.4 & 8.373 & 111.5 & 0.004 & 0.1 & 0.020396 \\
\hline K123 & 8.07 & 121.9 & 8.102 & 122.1 & 0.032 & 0.2 & 0.051225 \\
\hline A126 & 7.441 & 119 & 7.441 & 119 & 0 & 0 & 0 \\
\hline K131 & 7.42 & 117.2 & 7.449 & 117.1 & 0.029 & 0.1 & 0.035228 \\
\hline K132 & 7.346 & 119.3 & 7.302 & 119.3 & 0.044 & 0 & 0.044 \\
\hline A133 & 8 & 121.9 & 7.974 & 121.7 & 0.026 & 0.2 & 0.047707 \\
\hline A134 & 7.325 & 120.9 & 7.371 & 120.8 & 0.046 & 0.1 & 0.05016 \\
\hline A135 & 7.506 & 121 & 7.521 & 121.1 & 0.015 & 0.1 & 0.025 \\
\hline L136 & 7.464 & 116.9 & 7.475 & 116.9 & 0.011 & 0 & 0.011 \\
\hline E137 & 8.05 & 115 & 8.106 & 114.9 & 0.056 & 0.1 & 0.059464 \\
\hline D139 & 9.23 & 121.2 & 9.236 & 121.3 & 0.006 & 0.1 & 0.020881 \\
\hline E140 & 9.129 & 117.6 & 9.122 & 117.8 & 0.007 & 0.2 & 0.040608 \\
\hline N141 & 7.391 & 122 & 7.392 & 121.8 & 0.001 & 0.2 & 0.040012 \\
\hline F142 & 7.341 & 118.7 & 7.368 & 118.7 & 0.027 & 0 & 0.027 \\
\hline A148 & 7.191 & 122.7 & 7.19 & 122.6 & 0.001 & 0.1 & 0.020025 \\
\hline G153 & 8.154 & 107.7 & 8.154 & 107.7 & 0 & 0 & 0 \\
\hline G157 & 7.979 & 107.4 & 7.979 & 107.4 & 0 & 0 & 0 \\
\hline W158 & 7.921 & 124.5 & 7.93 & 124.4 & 0.009 & 0.1 & 0.021932 \\
\hline K159 & 8.579 & 118.4 & 8.566 & 118.2 & 0.013 & 0.2 & 0.042059 \\
\hline S160 & 8.303 & 113 & 8.314 & 113.1 & 0.011 & 0.1 & 0.022825 \\
\hline F161 & 7.565 & 123.5 & 7.567 & 123.6 & 0.002 & 0.1 & 0.0201 \\
\hline V162 & 8.69 & 118.5 & 8.67 & 118.3 & 0.02 & 0.2 & 0.044721 \\
\hline A163 & 7.69 & 120.5 & 7.7 & 121 & 0.01 & 0.5 & 0.100499 \\
\hline I164 & 7.054 & 117.4 & 7.059 & 117.4 & 0.005 & 0 & 0.005 \\
\hline D166 & 8.861 & 116.4 & 8.847 & 116.4 & 0.014 & 0 & 0.014 \\
\hline G167 & 7.671 & 106.9 & 7.671 & 106.9 & 0 & 0 & 0 \\
\hline I168 & 7.026 & 120.3 & 7.015 & 120.6 & 0.011 & 0.3 & 0.061 \\
\hline E169 & 8.801 & 128.4 & 8.831 & 128.8 & 0.03 & 0.4 & 0.08544 \\
\hline L170 & 7.929 & 124.8 & 7.952 & 124.7 & 0.023 & 0.1 & 0.03048 \\
\hline N171 & 9.623 & 121.5 & 9.631 & 121.4 & 0.008 & 0.1 & 0.021541 \\
\hline E172 & 8.584 & 117.1 & 8.567 & 117 & 0.017 & 0.1 & 0.026249 \\
\hline E174 & 8.7 & 120.9 & 8.628 & 121 & 0.072 & 0.1 & 0.074726 \\
\hline E175 & 8.735 & 119.4 & 8.741 & 119.4 & 0.006 & 0 & 0.006 \\
\hline
\end{tabular}




\begin{tabular}{|c|c|c|c|c|c|c|c|}
\hline R176 & 7.63 & 119.5 & 7.606 & 119.3 & 0.024 & 0.2 & 0.046648 \\
\hline L177 & 8.141 & 122.3 & 8.157 & 122.1 & 0.016 & 0.2 & 0.043081 \\
\hline A178 & 8.424 & 124.1 & 8.406 & 124 & 0.018 & 0.1 & 0.026907 \\
\hline A179 & 7.491 & 119 & 7.491 & 119 & 0 & 0 & 0 \\
\hline K180 & 8.266 & 122.2 & 8.251 & 122.1 & 0.015 & 0.1 & 0.025 \\
\hline G181 & 9.031 & 106.9 & 9.031 & 106.9 & 0 & 0 & 0 \\
\hline A182 & 7.876 & 121.2 & 7.875 & 121.1 & 0.001 & 0.1 & 0.020025 \\
\hline S183 & 8.015 & 113.2 & 8.005 & 112.9 & 0.01 & 0.3 & 0.060828 \\
\hline D184 & 9.236 & 121.8 & 9.249 & 121.8 & 0.013 & 0 & 0.013 \\
\hline A185 & 7.886 & 126 & 7.886 & 126 & 0 & 0 & 0 \\
\hline N187 & 8.18 & 117.4 & 8.178 & 117.3 & 0.002 & 0.1 & 0.0201 \\
\hline R188 & 8.823 & 122.1 & 8.835 & 121.7 & 0.012 & 0.4 & 0.080895 \\
\hline F189 & 8.399 & 120 & 8.385 & 120.2 & 0.014 & 0.2 & 0.042379 \\
\hline G190 & 8.055 & 104 & 8.055 & 104 & 0 & 0 & 0 \\
\hline L192 & 8.548 & 121.5 & 8.517 & 122 & 0.031 & 0.5 & 0.104695 \\
\hline L193 & 8.237 & 119.9 & 8.241 & 119.8 & 0.004 & 0.1 & 0.020396 \\
\hline R195 & 7.879 & 118.2 & 7.878 & 118.2 & 0.001 & 0 & 0.001 \\
\hline T196 & 9.018 & 111.1 & 8.984 & 111.1 & 0.034 & 0 & 0.034 \\
\hline F197 & 8.049 & 120 & 8.03 & 119.9 & 0.019 & 0.1 & 0.027586 \\
\hline A198 & 7.161 & 129.4 & 7.161 & 129.4 & 0 & 0 & 0 \\
\hline
\end{tabular}


